University of South Carolina

Scholar Commons

6-2015

\title{
Unmanned Aircraft Systems: Air-Ground Channel Characterization for Future Applications
}

David W. Matolak

University of South Carolina - Columbia, matolak@cec.sc.edu

Ruoyu Sun

Follow this and additional works at: https://scholarcommons.sc.edu/elct_facpub

Part of the Signal Processing Commons, Systems and Communications Commons, and the VLSI and Circuits, Embedded and Hardware Systems Commons

\section{Publication Info}

Postprint version. Published in IEEE Vehicular Technology Magazine, Volume 10, Issue 2, 2015, pages 79-85.

(C) IEEE Vehicular Technology Magazine, 2015, IEEE Matolak, D., Sun, R. (2015). Unmanned Aircraft Systems: Air-Ground Channel Characterization for Future Applications. IEEE Vehicular Technology Magazine, 10(2), 79-85.

http://dx.doi.org/10.1109/MVT.2015.2411191

This Article is brought to you by the Electrical Engineering, Department of at Scholar Commons. It has been accepted for inclusion in Faculty Publications by an authorized administrator of Scholar Commons. For more information, please contact digres@mailbox.sc.edu. 


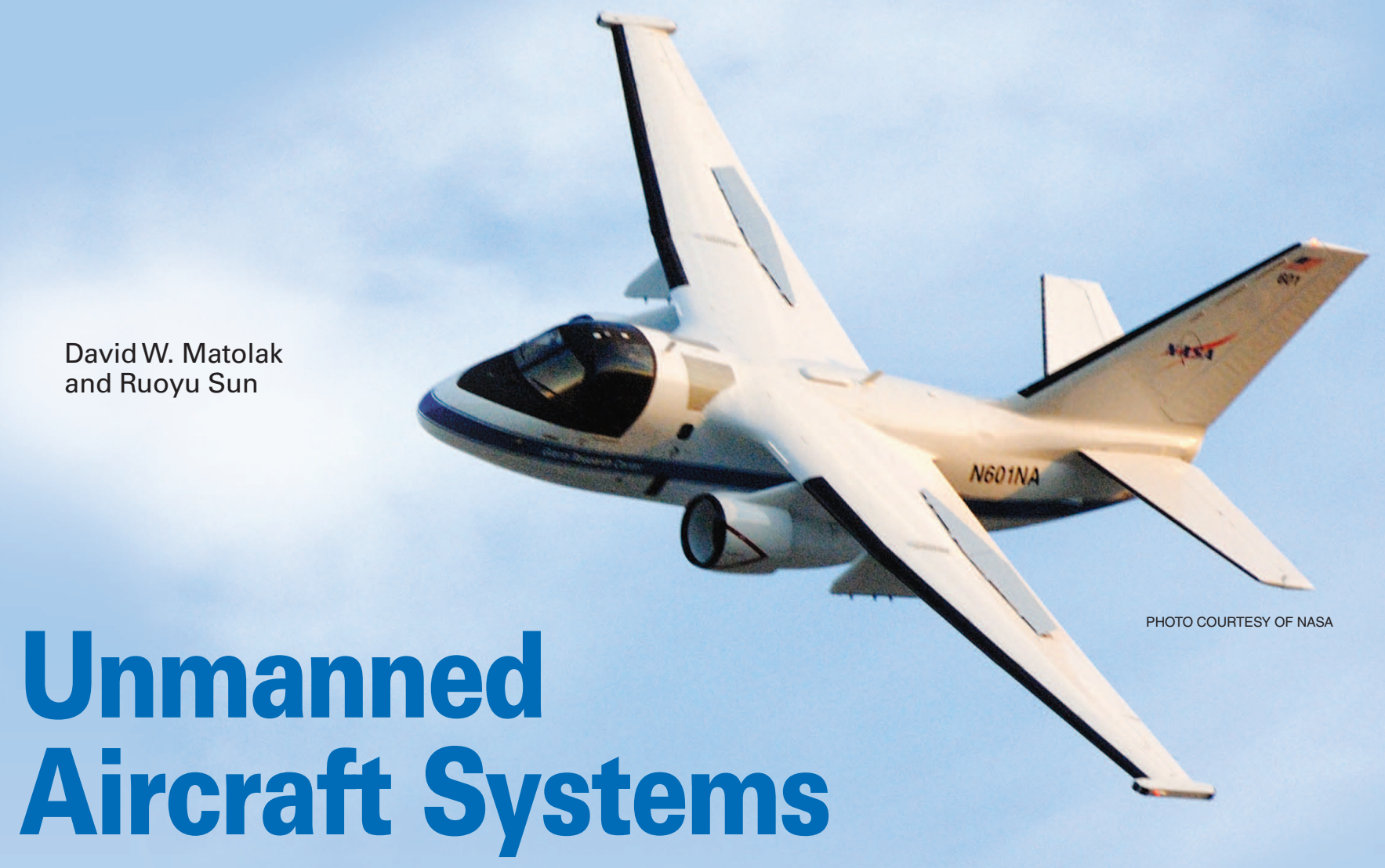

\section{Air-Ground Channel Characterization for Future Applications}

U nmanned aircraft systems (UASs) are being used increasingly worldwide. These systems will operate in conditions that differ from conventional piloted aircraft, and this implies that the airground (AG) channel for UASs can differ significantly from the traditional, simple, AG channel models. After providing some background and motivation, we describe the AG channel features and our efforts in measuring and modeling the AG channel. Some example measurement and model results-for the path loss and the Ricean $K$-factor-are provided to illustrate some of the interesting AG channel characteristics that are still being investigated.

The use of UASs is growing rapidly. These aircraft, also known as unmanned aerial vehicles (UAVs) and in the popular press by the misnomer drones, are being used for an

Digital Object Identifier 10.1109/MVT.2015.2411191

Date of publication: 19 May 2015 ever-increasing number of applications, including law enforcement, filmmaking, search and rescue, and industrial and scientific applications. Additional applications will invariably arise as these aircraft become less expensive and easier to deploy by nonexperts. Since the reliability and safety of UASs are paramount, strict requirements on the UAS communication link performance will be mandatory. It is well known that the wireless channel can be a significant impediment to reliable communication, and this is certainly true for the three-dimensional AG channel. Although a number of past efforts have been devoted to AG channel characterization, most of these were for fairly benign conditions with a tall ground site (GS) tower in a wide open (uncluttered) area, and for narrowband signals. Since the future UASs will not always operate in these conditions, new research on the AG channel-and models for it-are required for UAS applications. In this article, after providing a brief background and motivation, we provide a description of our work on AG channel 


\section{THE FAA HAS PARTNERED WITH NASA TO LEAD THE INVESTIGATION ON THE TECHNICAL CHALLENGES ASSOCIATED WITH UAS INTEGRATION.}

characterization for future UAS applications, including example measurement and modeling results.

\section{Preparing for UASs}

A recent report by the United States Department of Transportation [1] predicts that the number of UASs in the United States will increase from a few hundred in 2015 to over 230,000 in 2035 . The report contends that the majority of these UASs will be small and microvehicles, yet even a small percentage of this number implies a significant impact on airspace operations worldwide. The rapid expected growth in UAS use has incited many organizations to work on the various technical challenges that must be overcome to ensure the safe and reliable integration of UASs into the worldwide airspace.

In the United States, the governing body responsible for civil aviation is the Federal Aviation Administration (FAA). The FAA has been charged by the U.S. Congress to integrate UAS into the National Airspace System starting in 2015. Since the FAA is responsible for civil aviation safety, policy, and facilities engineering, but does not itself conduct research and development, it has partnered with other organizations to conduct the work necessary for UAS integration.

One of these organizations is the Radio Technical Commission for Aeronautics (RTCA) [2], the standards body responsible for U.S. civil aviation. The RTCA special committee (SC)-228 has been charged with the development of requirements for UAS control and nonpayload communications (CNPC); another SC is responsible for UAS detect-and-avoid standards. The RTCA members include representatives from the industry, academia, and government. The RTCA efforts provide input to the International Civil Aviation Organization (ICAO) [3], and the ICAO in turn provides input to the International Telecommunications Union [4]. The FAA has also established six test sites across the United States for authorized flight testing of UASs [5].

The FAA has partnered with the U.S. National Aeronautics and Space Administration (NASA) to lead the investigation on the technical challenges associated with UAS integration. The NASA program is known as $U A S$ Integration in the National Airspace System [6]. We are working with NASA's John H. Glenn Research Center on a project that is characterizing the AG channel, evaluating the performance of potential CNPC waveforms over the AG channels, and developing simulations for aeronautical networking that incorporate UASs along with piloted aircraft.

\section{AG Channel Characteristics and Modeling}

A moderate body of literature exists for the AG channel, going back over the past 60 years; see [7] for a comprehensive literature review. Despite this long history, the number of papers in the literature on the AG channel is far smaller than the number on other types of channels such as cellular radio. The available AG channel studies have predominantly addressed very narrowband channels for single-antenna systems (at each link end) and at disparate frequencies in a small number of representative environments. Only recently has this research void begun to be addressed [8].

\section{Basic AG Channel Characteristics}

The AG channel will often, but not always, contain a line-ofsight (LOS) component. Shadowing may occur due to (Earth) surface-based obstacles, such as buildings, terrain, or trees but can also occur from the aircraft itself during flight maneuvers; the latter type of shadowing has received only scant attention [9]. Multipath components (MPCs) occur primarily from surface-based obstacles (although MPCs can arise from the aircraft itself, these are typically weak and have a very small relative delay compared to MPCs from surface obstacles), and their number and relative strength depends critically on the environment surrounding the GS and in general within the (ellipsoidal) volume between the GS and aircraft (as foci). In most conditions, the primary MPC will be that of the surface reflection. This has led to what might be termed the canonical model for the AG channel: the two-ray model with one LOS component (ray) and one surface reflection. For most terrestrial applications, the two-ray model assumes a flat Earth, but this can be inaccurate for AG applications, particularly when the link distances exceed a few tens of kilometers. This then requires the more complex curved-Earth two-ray (CE2R) model, the geometry of which is shown in Figure 1. An analysis of this model appears in [11] and is included in a journal paper that has been submitted for publication. The CE2R model we have developed accounts for surface electrical characteristics, spherical wave divergence, and surface roughness. Additional considerations, such as ducting, foliage attenuation, atmospheric gas attenuation, and hydrometeor attenuations, were discussed in [11]. These effects occur with a small probability and/or have a minor impact on the AG channel in our bands.

Regarding spectral allocations, for UASs, these have been established in the $L$-band $(\sim 1-2 \mathrm{GHz})$ and in the $C$-band (4-8 GHz), but since many other systems (aviation and otherwise) operate in these bands, the actual available spectrum is limited: there is approximately $17 \mathrm{MHz}$ (960-977 MHz) at the $L$-band and $61 \mathrm{MHz}(5.03-5.091 \mathrm{GHz})$ at the $C$-band presently allocated for UAS CNPC. This limited spectrum-particularly at the $L$-band-presents significant challenges to the design of a high-capacity CNPC network. The basic propagation conditions can also 
differ significantly between the two bands. For example, free-space path loss is approximately $14 \mathrm{~dB}$ larger in the $C$-band than in the $L$-band. The factor of five wavelength difference also means that reflecting surfaces are considerably smoother at the $L$-band than at the $C$-band. This has implications for the strength of MPCs, which can produce a substantial distortion of the transmitted signals.

Finally, on these basic AG channel characteristics, since UASs do not have to accommodate humans within the aircraft, UAS flight dynamics are not required to be as gentle as those of human-occupied aircraft. Even with the FAA restrictions on allowed UAS airspace volumes, small UASs especially may employ highly dynamic flight paths and may operate at low elevation angles and low altitudes-much nearer to terrestrial obstacles such as buildings and trees-than conventional aircraft. This also has direct implications for the AG channel MPC characteristics. In summary, UAS AG channels will often be more dispersive, incur larger terrestrial shadowing attenuations, and change more rapidly due to flight maneuvers than the channels incurred by conventional aircraft.

\section{AG Channel Modeling}

The two-ray models, along with the simple free-space path loss model (which neglects any surface reflection) are simple analytical models. The full CE2R model path loss in decibels is given by

$$
L_{p}=20 \log [4 \pi d / \lambda]-20 \log \left\{\left|1+r D \Gamma_{p} \exp (-j 2 \pi \Delta R / \lambda)\right|\right\},
$$

where $d$ is the link distance, $\lambda$ is the wavelength, $r$ is the surface-roughness factor, $D$ is the divergence factor due to the spherical Earth, $\Gamma$ is the surface reflection coefficient, with the subscript $p$ denoting impinging wave polarization, and $\Delta R$ is the relative path length difference between the LOS and surface reflection. Depending on the surface type (e.g., ground or water), $r$ typically assumes a Gaussian distribution of surface height and, for water surfaces, is related to the wind speed. The roughness factor $r$ also depends on the wavelength and the grazing angle ( $\psi_{2}^{\prime}$ in Figure 1), computed from geometry. The divergence factor $D$ is computed from geometry [11], and the reflection coefficient $\Gamma$ requires the electrical parameters of the surface as well as the grazing angle. Note that antenna gains can also be incorporated into both the LOS and reflected components.

The two-ray model is, of course, inaccurate (or at least incomplete) for settings where additional MPCs may be present. We have found this to be true even for over-water settings, in which obstacles on the water surface (e.g., boats and drilling platforms) and large ocean waves can induce intermittent MPCs, rendering the over-water AG channel a sparse multipath channel.

Classical narrowband path loss models (e.g., Longley-Rice) require path profiles, typically only predict

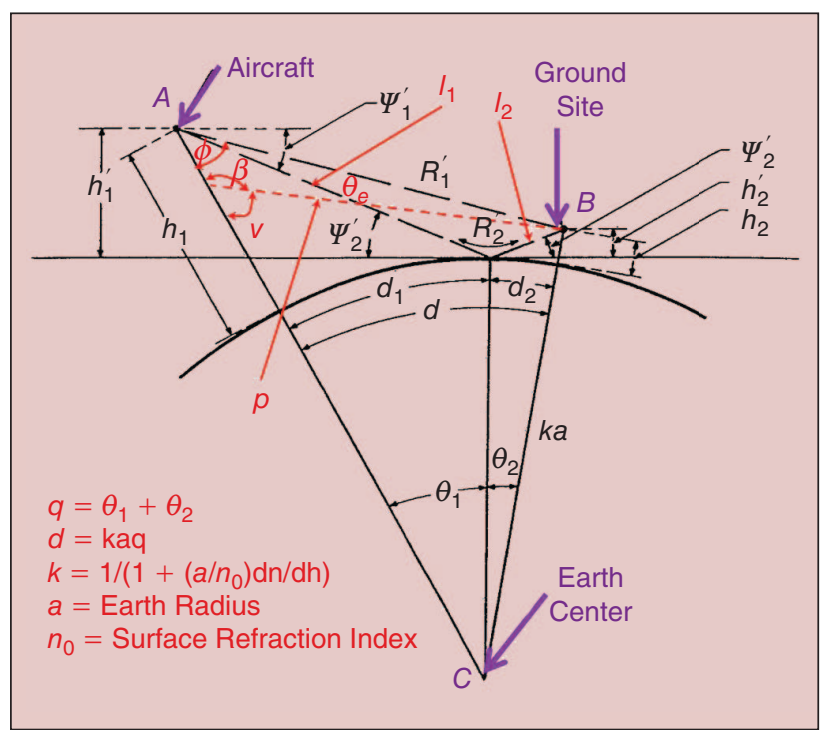

FIGURE 1 The CE2R geometry (adapted from [10]).

median attenuation, and are also incomplete for wideband channel characterization. Similarly, although satellite (to ground) channels share much in common with the AG channel (aside from any ionospheric or other high-altitude atmospheric effects), these too have largely focused on narrowband cases.

Deterministic models, such as those using a high-frequency approximation and ray tracing, can be employed for the AG channel, but these typically require a large database to describe the local environment and are, hence, computationally intensive especially for complex environments. They also do not model diffuse scattering. The more recent geometry-based stochastic channel models (GBSCMs), e.g., [12], offer a promising compromise between the large computations of ray tracing and the more traditional statistical models by randomizing obstacle placement (based on measurements) and incorporating diffuse scattering components.

Our models for the AG channel are still evolving, but for the simplest over-water settings, we have arrived at a quasi-deterministic model that consists of the CE2R model plus random intermittent MPCs. For other settings for which we have measured data-including suburban, hilly, near-urban, desert, and mountainous terrain-more complex models will be required, but, except in cases where the LOS and/or surface reflection is blocked, the CE2R model will still form the AG channel model foundation.

Some final remarks on the topic of modeling are that the set of environments for which we are developing models is unlikely to be complete, and the channel classification problem will arise when one attempts to apply any empirically based models to GS environments that may differ from those in which the measurements were made. The development of AG GBSCMs can alleviate this. Purely stochastic models, such as the traditional 
SINCE UASS DO NOT HAVE TO ACCOMMODATE HUMANS WITHIN THE AIRCRAFT, UAS FLIGHT DYNAMICS ARE NOT REQUIRED TO BE AS GENTLE AS THOSE OF HUMAN-OCCUPIED AIRCRAFT.

tapped-delay line, are computationally efficient but require careful construction and parameterization to account for changing channel statistics over flight paths (so-called nonstationary models).

\section{AG Channel Measurement Campaign}

Flight test measurements are expensive and time-consuming, but to establish a database from which empirical models can be developed and against which analytical and simulation models can be validated, we have conducted AG channel measurement flights. In our NASA project, we have made measurements in the two

\begin{tabular}{llll}
\hline TABLE & The channel sounder parameters. \\
Band & $\begin{array}{l}\text { Signal Band- } \\
\text { width (MHz) }\end{array}$ & $\begin{array}{l}\text { Frequency } \\
\text { Span }(\mathrm{MHz})\end{array}$ & $\begin{array}{l}\text { Maximum Delay } \\
\text { Span }(\mu \mathrm{s})\end{array}$ \\
\hline L & 5 & $960-977$ & 204.6 \\
C & 50 & $5,000-5,150$ & 20.46 \\
\hline
\end{tabular}

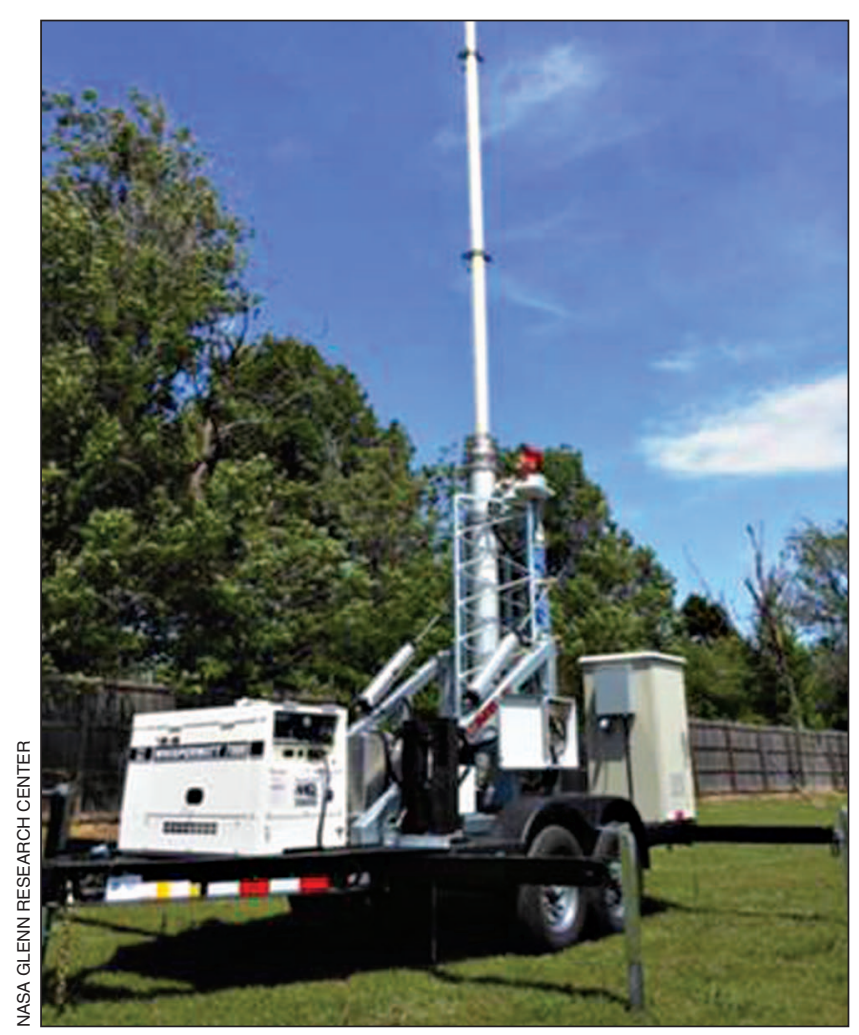

FIGURE 2 The transportable tower and GS. frequency bands allocated for UASs. The measurements were made with a dual-band direct-sequence spread spectrum stepped correlator single-input/multiple-output channel sounder that transmitted a signal in each band simultaneously and that was received by two antennas in each band. The measurement outputs are power delay profiles (PDPs) for each of the four receivers (Rxs). Some channel sounder specifications appear in Table 1. The maximum PDP output rate is approximately 3,000 PDPs/s.

Figure 2 shows the GS, and Figure 3 shows the location of the four Rx antennas under NASA's (piloted) S-3B aircraft. The aircraft antennas are monopoles mounted on the aircraft underside in a rectangular pattern $(\sim 1.3 \mathrm{~m} \times 1.4 \mathrm{~m})$. These antennas are nearly omnidirectional in azimuth with a gain of $5 \mathrm{~dB}$. The GS antennas have gains of $6 \mathrm{~dB}$ for the $C$-band, $5 \mathrm{~dB}$ for the $L$-band, and elevation/azimuth beamwidths of approximately $35 / 180^{\circ}$ for the $C$-band and $60 / 120^{\circ}$ for the $L$-band. The transmitter power for both bands was $40 \mathrm{dBm}$, and the $C$-band transmitter employed an external high-power amplifier of gain $7 \mathrm{~dB}$. The Rxs employed external low-noise amplifiers of gain $30 \mathrm{~dB}$ in the $C$-band and $15.5 \mathrm{~dB}$ in the $L$-band.

Example flight tracks (in the Google Maps view) are shown in Figure 4 for flights over the desert and near the mountains, with the GS in Palmdale, California. Both straight and oval-shaped flight tracks were flown to vary the orientation from the GS to the aircraft antennas. The different colors denote different flights or different segments of measurement files. Similar flight tracks, typically all at a constant altitude, which ranged from approximately 500 to $2,000 \mathrm{~m}$, were flown in the other GS environments. Figure 5 shows a view from the GS location for flight tests conducted near Cleveland, Ohio, with the urban city center to the east.

The channel characteristics obtained from the measurements include the propagation path loss, delay spreads, Doppler characteristics, small-scale fading characteristics,

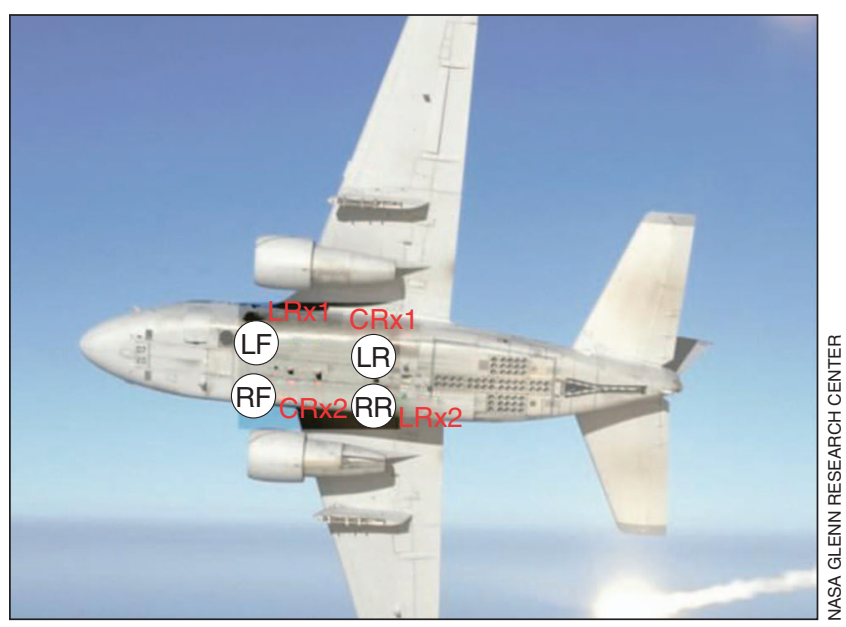

Figure 3 A view of the underside of the S-3B aircraft. 
intermittent MPC statistics, and correlations among the signals received on the four antennas.

\section{Example AG Channel Results and Models}

The example path loss versus distance results are shown in Figure 6 for the suburban/hilly terrain environment near Latrobe, Pennsylvania. Both the measured and CE2R model results for the $L$-band are shown. The model results also include Ricean fading (described subsequently). The path loss values larger than the CE2R model at short link distances are attributed to the aircraft antenna gain at these higher elevation angles.

A sequence of PDPs for a flight track segment in this suburban/hilly environment is shown in Figure 7. The MPCs present are attributable to several large buildings in the town of Latrobe and, to a lesser degree, the ridge running parallel to the flight track from northeast to southwest. The root-mean square delay spreads range from 42 to $600 \mathrm{~ns}$.

To compute the statistics for a small-scale fading analysis, one must determine the region of space over which the channel can be assumed statistically stationary. We have estimated the stationarity distance for the over-water AG channels using a temporal PDP correlation coefficient [13] $c\left(\Delta t, t_{i}\right)$

$$
c\left(\Delta t, t_{i}\right)=\frac{\int P_{\mathrm{avg}, N}\left(\tau, t_{i}\right) P_{\mathrm{avg}, N}\left(\tau, t_{i}+\Delta t\right) d \tau}{\max \left\{\int\left[P_{\mathrm{avg}, N}\left(\tau, t_{i}\right)\right]^{2} d \tau, \int\left[P_{\mathrm{avg}, N}\left(\tau, t_{i}+\Delta t\right)\right]^{2} d \tau\right\}} .
$$

This metric quantifies how similar the average PDP at time $t_{i}\left(P_{\mathrm{avg}, N}\left(\tau, t_{i}\right)\right)$ is to the average PDP at time $t_{i}+\Delta t$; this is also a function of the starting time $t_{i}$. The variable $\tau$ is the delay, and the PDP is averaged over a small window to remove any rapid small-scale fading and equipment variations. With the known aircraft velocity $v$, we can compute the distance as $\Delta x=v \Delta t$.

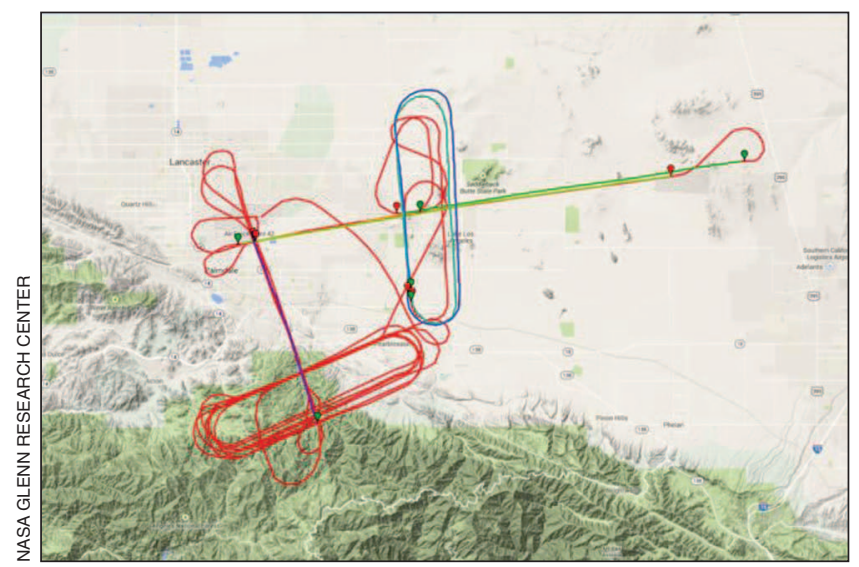

FigURE 4 The example flight tracks (Google Maps view) for desert and mountainous terrain.
TO COMPUTE THE STATISTICS FOR A SMALL-SCALE FADING ANALYSIS, ONE MUST DETERMINE THE REGION OF SPACE OVER WHICH THE CHANNEL CAN BE ASSUMED STATISTICALLY STATIONARY.

We declare the channel stationary for the range of distance values $(\Delta x)$ for which this correlation coefficient remains above the value 0.9 . With this stationarity distance ( $\sim 250 \lambda$ at the $C$-band), we have computed statistics on the correlation between the LOS components received on all four antennas and the Ricean $K$-factor for all four antennas. For brevity, we report here only the $K$-factor results.

We have computed the Ricean $K$-factor over the stationarity distance for several environments and for both bands using two methods, a maximum-likelihood method $\left(K_{\mathrm{ML}}\right)$ and a method that employs second and fourth moments $\left(K_{2,4}\right)$. The results with the two methods are nearly identical. Figures 8 and 9 show the results for $K$ versus the link distance computed for the suburban/hilly terrain. Table 2 provides some maximum-likelihood $K$-factor statistics, where our linear fit to the $K$-factor (in decibels) versus the distance $d$ in kilometers is given by the equation

$$
K(d)=A+n\left(d-d_{\min }\right)+X,
$$

where $A$ is a constant value for the minimum distance $d_{\text {min }}$ (2.2 km for the $C$-band, $1.2 \mathrm{~km}$ for the $L$-band), $n$ is the slope, and $X$ is a zero-mean Gaussian random variable with the standard deviation $\sigma_{X}$. The values for $K$ in Table 2 are valid from the minimum distance up to $17.5 \mathrm{~km}$.

Interestingly, the $K$-factor is larger for the $C$-band than for the $L$-band. We attribute this to the stronger surface reflection(s) at the $L$-band (since the surface is smoother at the longer wavelength). In both bands, $K$ increases slightly with distance.

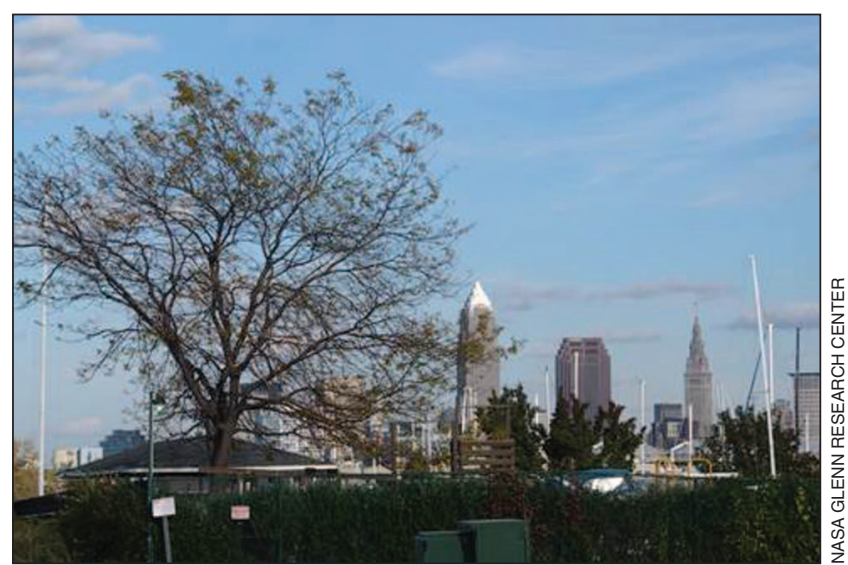

FIGURE 5 A view from the GS (looking east) toward downtown Cleveland, Ohio. 


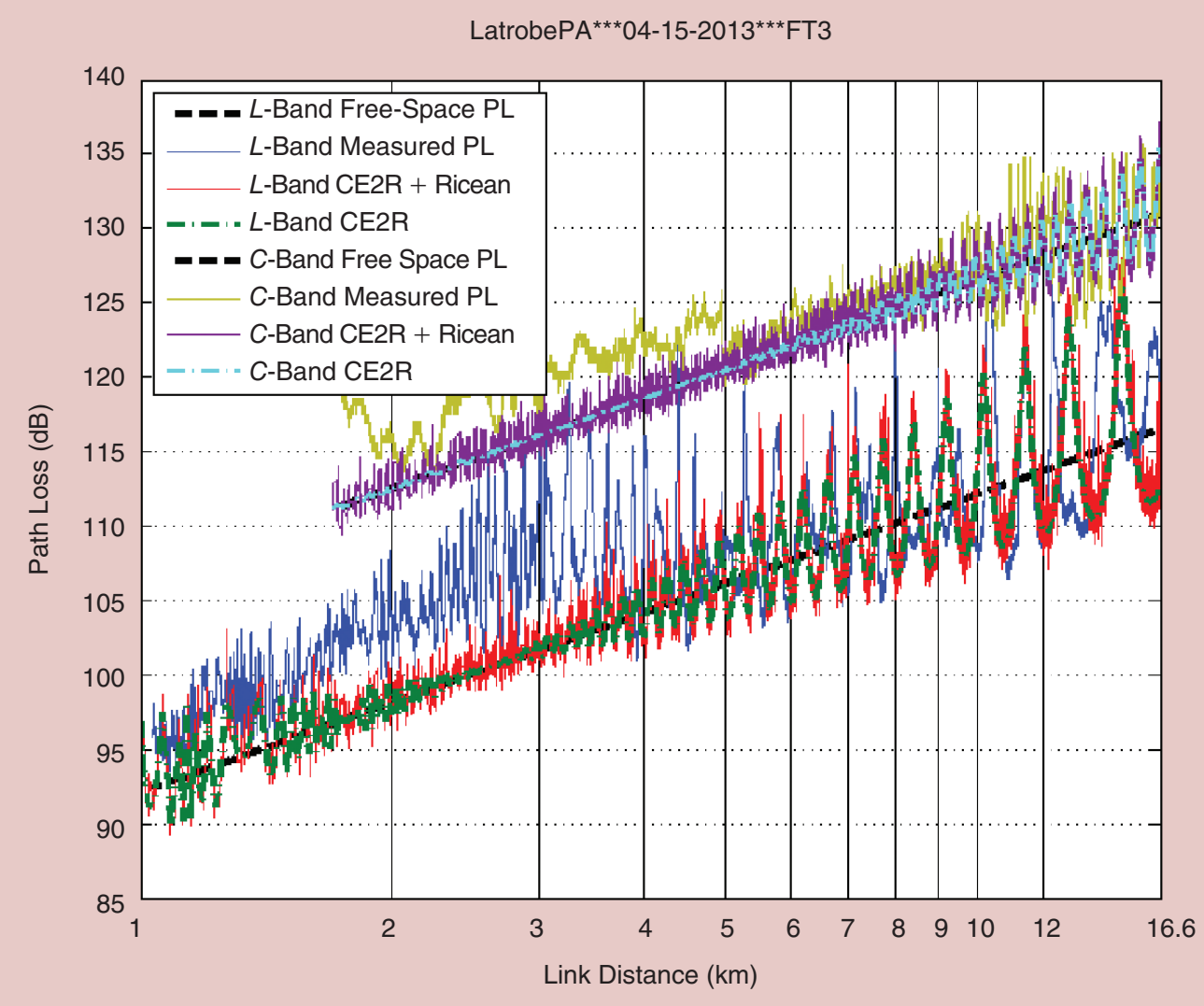

FIGURE 6 The path loss versus the distance for suburban/hilly terrain in the L-band.

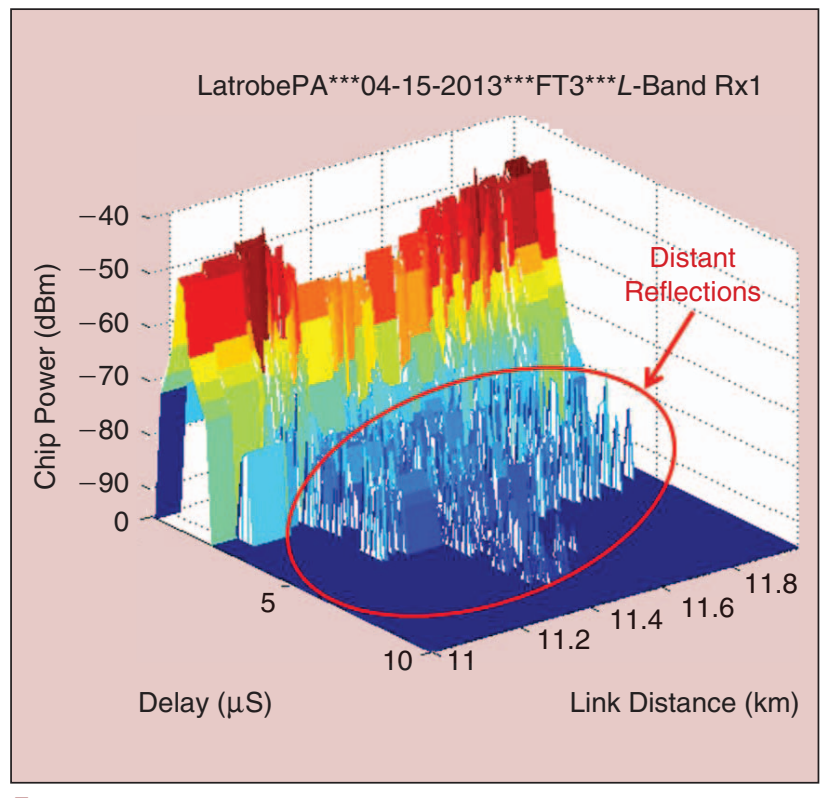

FIGURE 7 The sequence of PDPs versus the distance for suburban/ hilly terrain in the $L$-band.

\section{Summary and Future Work}

In this article, we have provided motivation to study the channel characteristics for the AG channel for future UAS applications. Although the AG channel has

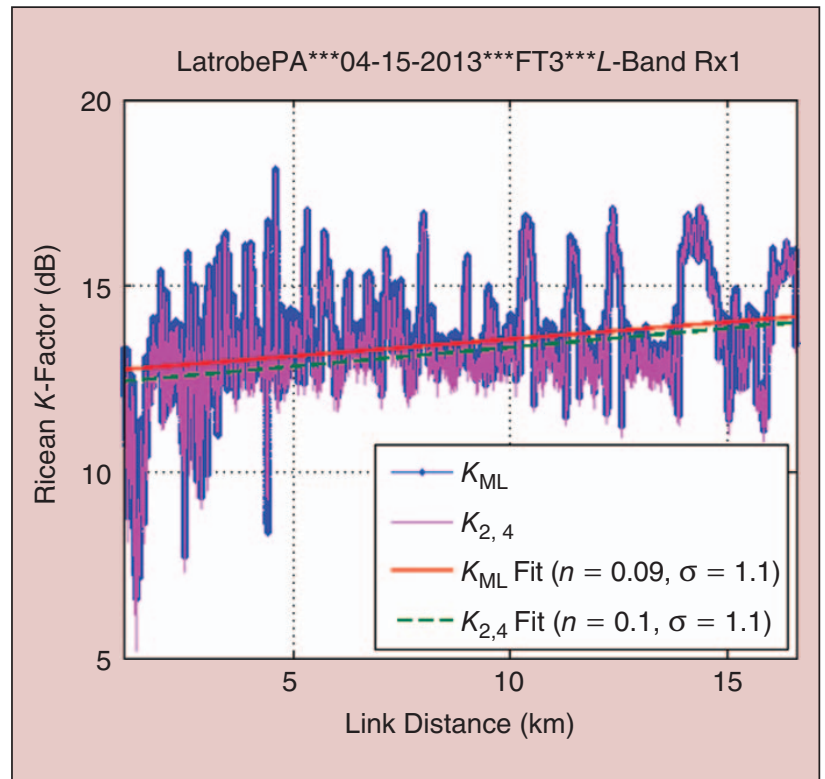

FIGURE 8 The example Ricean $K$-factor versus the distance in the $C$-band for suburban/hilly terrain.

been studied for some time, the past studies were incomplete for the new UAS applications. The basic AG channel characteristics were described along with the limitations of the existing models. We then described 


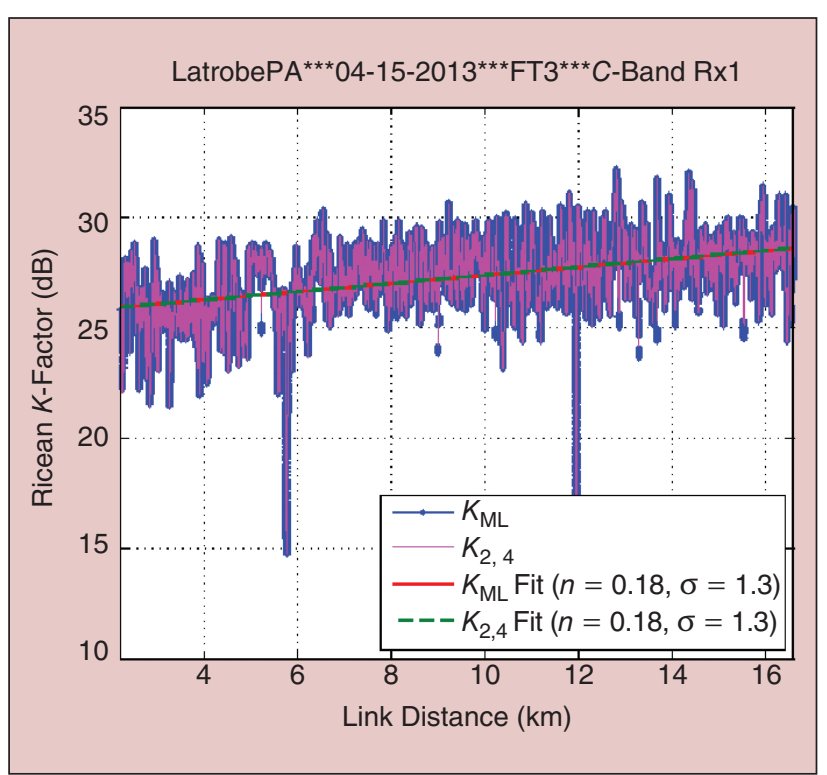

FigurE 9 The example Ricean $K$-factor versus the distance in the $L$-band for suburban/hilly terrain.

TABLE 2 The maximum-likelihood $K$-factor statistics and linear fit parameters for a straight flight track, suburban/hilly terrain.

\begin{tabular}{llllll} 
& & \multicolumn{2}{c}{ C-Band } & \multicolumn{2}{c}{ L-Band } \\
& & $\boldsymbol{R x}$ 1 & $\boldsymbol{R x 2}$ & $\boldsymbol{R x}$ 1 & $\boldsymbol{R x 2}$ \\
\hline Linear Fit & $A(\mathrm{~dB})$ & 25.5 & 25.6 & 12.7 & 13.4 \\
Parameters & $n$ & 0.19 & 0.16 & 0.09 & 0.10 \\
& $\sigma_{x}(\mathrm{~dB})$ & 1.4 & 1.4 & 1.1 & 0.9 \\
Statistics & Maximum & 32.2 & 32.4 & 18.1 & 18.9 \\
(dB) & Minimum & 13.7 & 14.5 & 6.5 & 6.5 \\
& Median & 27.3 & 27.0 & 13.5 & 14.3 \\
& Mean & 27.5 & 27.2 & 13.3 & 14.2 \\
& Standard & 1.6 & 1.5 & 1.2 & 1.0 \\
& deviation & & & & \\
\hline
\end{tabular}

our AG channel measurement campaign and provided example measurement and model results for propagation path loss and the Ricean $K$-factor in a suburban/ hilly environment.

Future work includes the development of complete statistical models for the AG channel in all measurement environments. The use of geometry-based models, particularly GBSCMs, will also be investigated.

\section{Acknowledgments}

We would like to acknowledge the generous support from NASA and, in particular, the efforts of Glenn Research Center Engineers J. Griner, K. Shalkhauser, and R. Kerczewski, whose work has enabled the progress reported here.

\section{Author Information}

David W. Matolak (matolak@sc.edu) received his B.S., M.S., and Ph.D. degrees from Pennsylvania State University, the University of Massachusetts, and the University of Virginia, respectively, all in electrical engineering. He has over 20 years of experience in research, development, design, and deployment in civil and military terrestrial, aeronautical, and satellite communication systems. His prior employers include AT\&T Bell Labs, L3 Communication Systems, MITRE, Lockheed Martin, and Ohio University. He has over 100 publications and eight patents, and his research interests are radio channel modeling and communication techniques for nonstationary fading channels, multicarrier transmission, and mobile ad hoc networks.

Ruoyu Sun (sun55@email.sc.edu) received his B.S. degree from Tianjin University, China, in 2004 and his M.S. degree from Beijing Jiaotong University, China, in 2007 , both in electrical engineering. He is currently working toward the Ph.D. degree in the Department of Electrical Engineering at the University of South Carolina. He was employed by T3G Technology Co., Ltd. and Motorola, Inc., all in Beijing, China. His research interests include wireless channel measurements and channel modeling.

\section{References}

[1] US Dept. Transportation, "Unmanned aircraft system (UAS) service demand 2015-2035: Literature review and projections of future usage," Tech. Rep. v.1.0, DOT-VNTSC-DoD-13-01, Feb. 2014.

[2] (2014, Oct. 31). Radio technical commission for aeronautics. [Online]. Available: www.rtca.org

[3] (2014, Oct. 31). International civil aviation organization. [Online]. Available: www.icao.int

[4] (2014, Oct. 31). International telecommunications union. [Online]. Available: www.itu.int

[5] D. Werner, "Preparing for the era of unmanned planes," in Proc. AIAA Aerospace America, Sept. 2014, pp. 38-42.

[6] (2014, Oct. 31). National Aeronautics and Space Administration. [Online]. Available: http://www.aeronautics.nasa.gov/isrp/uas/index. htm

[7] D. W. Matolak, "Air-ground channels and models: Comprehensive review and considerations for unmanned aircraft systems," in Proc. IEEE Aerospace Conf., Big Sky, MT, Mar. 3-10, 2012, pp. 1-17.

[8] M. Simunek, F. P. Fontan, and P. Pechac, "The UAV low elevation propagation channel in Urban areas: Statistical analysis and timeseries generator," IEEE Trans. Antennas Propagat., vol. 61, no. 7, pp. $3850-3858$, July 2013.

[9] J. Kunisch, I. de la Torre, A. Winkelman, M. Eube, and T. Fuss, "Wideband time-variant air-to-ground radio channel measurements at $5 \mathrm{GHz}$," in Proc. 5th European Conf. Antennas Propagation, Rome, Italy, Apr. 11-15, 2011, pp. 1386-1390.

[10] E. C. Jordan and K. G. Balmain, Electromagnetic Waves and Radiating Systems, 2nd ed. Englewood Cliffs, NJ: Prentice Hall, 1968.

[11] D. W. Matolak, "AG channel measurements and modeling: Initial analysis and flight test planning," NASA Glenn Research Center, Grant NNX12AD53G, Tech. Rep. 2, June 8, 2012.

[12] J. Karedal, F. Tufvesson, N. Czink, A. Paier, C. Dumard, T. Zemen, C. F. Mecklenbrauker, and A. F. Molisch, "A geometry-based stochastic MIMO model for vehicle-to-vehicle communications," IEEE Trans. Wireless Commun., vol. 8, no. 7, pp. 3646-3657, July 2009.

[13] A. Gehring, M. Steinbauer, I. Gaspard, and M. Grigat, "Empirical channel stationarity in urban environments," in Proc. 4th European Personal Mobile Communications Conf., Vienna, Austria, Feb. 20-22, 2001, pp. 1-6. 